\title{
INTEGRAL observation of the high-mass X-ray transient V $0332+53$ during the 2005 outburst decline
}

\author{
N. Mowlavi ${ }^{1,2}$, I. Kreykenbohm ${ }^{1,3}$, S. E. Shaw ${ }^{1,4}$, K. Pottschmidt ${ }^{5}$, J. Wilms ${ }^{6}$, J. Rodriguez ${ }^{7,1}$, \\ N. Produit ${ }^{1}$, S. Soldi ${ }^{1,2}$, S. Larsson ${ }^{8}$, and P. Dubath ${ }^{1,2}$
}

\author{
1 INTEGRAL Science Data Centre, Ch. d'Écogia, 1290 Versoix, Switzerland \\ 2 Observatoire de Genève, Ch. des Maillettes, 1290 Versoix, Switzerland \\ 3 Institut für Astronomie und Astrophysik - Astronomie, Sand 1, 72076 Tübingen, Germany \\ ${ }^{4}$ School of Physics and Astronomy, University of Southampton, SO171BJ, UK \\ 5 Center for Astrophysics and Space Sciences, University of California at San Diego, La Jolla, CA 92093-0424, USA \\ 6 Department of Physics, University of Warwick, Coventry, CV4 7AL, UK \\ 7 CEA Saclay, DSM/DAPNIA/SAp(CNRS UMR 7158 AIM), 91191 Gif-Sur-Yvette, France \\ 8 Stockholm Observatory, Alba Nova, 10691 Stockholm, Sweden
}

Received 21 September 2005 / Accepted 14 December 2005

\section{ABSTRACT}

The decline of the high mass X-ray transient V 0332+53 during the Dec. 2004 to Feb. 2005 outburst is analysed from the data recorded by INTEGRAL. The flux is shown to decrease exponentially until 2005 Feb. 10, with a decay time scale of $\sim 30$ days above $20 \mathrm{keV}$ and $\sim 20$ days at lower energies, and to decrease linearly thereafter.

The energy spectrum is well modelled throughout the decay by a power law with a folding energy of $\sim 7.5 \mathrm{keV}$, and with two cyclotron absorption features. The folding energy does not vary significantly over the decay, but the spectrum becomes harder with time. Most importantly, we show that the parameters describing the fundamental cyclotron line around $27 \mathrm{keV}$ do vary with time: its energy and depth increase (by about $17 \%$ for the energy in $\sim 6$ weeks), while its width decreases. These changes of the cyclotron line parameters are interpreted as resulting from a change in the extent of the cyclotron scattering region.

Two quasi-periodic oscillations are also observed at various times during the observations, one at $0.05 \mathrm{~Hz}$ and another one near the pulsation frequency around $0.23 \mathrm{~Hz}$.

Key words. X-ray: stars - stars: pulsars: individual: V0332+53 - stars: magnetic fields - X-ray: bursts

\section{Introduction}

The recurrent X-ray transient V 0332+53 experienced an X-ray outburst from December 2004 to February 2005. The outburst was predicted one year earlier from an optical brightening of the massive O8-9Ve companion star, BQ Cam (Goranskij \& Barsukova 2004) and was first detected by the Rossi X-ray Timing Explorer (RXTE) in November 2005 (Swank et al. 2004). The INTernational Gamma-Ray Astrophysics Laboratory (INTEGRAL) started to observe the source on 2005 Jan. 6 (Kreykenbohm et al. 2005, hereafter Paper I) and monitored its X-ray activity until the end of February.

The observation of this fourth detected outburst of $\mathrm{V} 0332+53$ in thirty years revealed the presence of three cyclotron lines (Coburn et al. 2005; Paper I; Pottschmidt et al. 2005). The fundamental has an energy of $28 \mathrm{keV}$, and the first and second harmonics are at $50 \mathrm{keV}$ and $71 \mathrm{keV}$, respectively. The magnetic field strength is $2.7 \times 10^{12} \mathrm{G}$ (Paper I, Pottschmidt et al. 2005). The spectrum is otherwise described by a power law with a high-energy cut-off, typical of the spectra of X-ray pulsars. A new Quasi Periodic Oscillation (QPO) around the $0.23 \mathrm{~Hz}$ spin frequency of the neutron star has also been reported in the RXTE data (Qu et al. 2005), in addition to the $0.05 \mathrm{~Hz}$ QPO discovered by Takeshima et al. (1994) in the Ginga data of the 1989 outburst.

In Paper I, we presented the INTEGRAL observation of $\mathrm{V} 0332+53$ around the outburst peak. This paper presents the evolution of $\mathrm{V} 0332+53$ during its decline phase. The observations and data analysis method are described in Sects. 2 and 3, respectively. We then present in Sect. 4 the evolution during the decline of the fluxes in several energy bands, of the energy spectrum, and of the variability spectrum of $\mathrm{V} 0332+53$. The results are then discussed in Sect. 5.

\section{Observations}

The INTEGRAL satellite carries three high energy instruments, the imager IBIS (operating in the range $15 \mathrm{keV}$ to $10 \mathrm{MeV}$ ), the spectrometer SPI (20 keV to $8 \mathrm{MeV}$ ) and two identical 
Table 1. Summary of observations. From first to last column: (1) Revolution and pointing numbers; (2) Earth date of the start of the observation in UTC / IJD (INTEGRAL Julian Day = MJD - 51544) / MJD (Modified Julian Day); (3) Earth date of the end of the observation in MJD; (4) Individual pointing duration; (5) Total IBIS observation time; (6) Sum of IBIS Good Time Intervals; (7) Total JEM-X1 observation time; (8) Sum of JEM-X1 Good Time Intervals.

\begin{tabular}{|c|c|c|c|c|c|c|c|}
\hline \multirow[b]{2}{*}{$\begin{array}{r}\text { Revolution / } \\
\text { pointings }\end{array}$} & \multirow[b]{2}{*}{$\begin{array}{c}\text { Start date } \\
\text { (UTC / IJD / MJD) }\end{array}$} & \multirow[b]{2}{*}{$\begin{array}{l}\text { End date } \\
\text { (MJD) }\end{array}$} & \multirow{2}{*}{$\begin{array}{c}\text { Pointing } \\
\text { duration } \\
\text { (s) }\end{array}$} & \multicolumn{2}{|c|}{ IBIS } & \multicolumn{2}{|c|}{ JEM-X1 } \\
\hline & & & & $\begin{array}{r}\text { Exposure } \\
(\mathrm{s})\end{array}$ & $\begin{array}{r}\Sigma \text { GTIs } \\
(\mathrm{s})\end{array}$ & $\begin{array}{r}\text { Exposure } \\
\text { (s) }\end{array}$ & $\begin{array}{r}\Sigma \text { GTIs } \\
(\mathrm{s})\end{array}$ \\
\hline $272 / 75$ & 2005-01-06T08:48/1832.36724/53376.36724 & 53376.76044 & 42184 & 42184 & 30308 & 36896 & 36446 \\
\hline $273 / 66-81$ & 2005-01-08T22:18/1834.92997/53378.92997 & 53379.65749 & 3500 & 60953 & 59586 & 60953 & 56693 \\
\hline 274/04-09 & 2005-01-10T03:20/1836.13991/53380.13991 & 53380.39113 & 3500 & 21119 & 20901 & 21119 & 19801 \\
\hline $278 / 48-74$ & 2005-01-23T16:39/1849.69471/53393.69471 & 53394.66610 & 2858 & 80901 & 58411 & 75190 & 73078 \\
\hline $284 / 04-45$ & 2005-02-09T01:15/1866.05280/53410.05280 & 53410.86456 & 3572 & 149086 & 147739 & 149086 & 147469 \\
\hline $285 / 02-08$ & 2005-02-12T01:02/1869.04402/53413.04402 & 53413.23174 & 2200 & 15517 & 15349 & 15517 & 14394 \\
\hline $286 / 18-24$ & 2005-02-15T10:41/1872.44588/53416.44588 & 53416.63218 & 2200 & 15403 & 15093 & 15403 & 15185 \\
\hline $287 / 02-08$ & 2005-02-18T00:37/1875.02612/53419.02612 & 53419.23077 & 2200 & 16988 & 16628 & 16988 & 15809 \\
\hline $288 / 02-08$ & 2005-02-21T00:25/1878.01820/53422.01820 & 53422.25899 & 2200 & 20101 & 19226 & 20101 & 18854 \\
\hline
\end{tabular}

X-ray instruments JEM-X1 and JEM-X2 (3 to $35 \mathrm{keV}$ ) operational in turn (see Winkler et al. 2003 for more information on the INTEGRAL mission and instruments). IBIS is further composed of two modules, ISGRI operational down to $15 \mathrm{keV}$ and PICsIT above $200 \mathrm{keV}$.

INTEGRAL observed V0332+53 from 2005 Jan. 6 to Feb. 21. Three main observation sets were scheduled, in revolutions 272-274 ( 120 ks), 278 ( $\sim 80 \mathrm{ks})$ and $284(\sim 150 \mathrm{ks})$, followed by four $\sim 15 \mathrm{ks}$ observations from revolution 285 to 288 to monitor the tail of the outburst decay. The observations are summarized in Table 1. Except for the observation in revolution 272 , which was performed in a staring mode, all observations were made following a hexagonal dithering pattern. The hexagonal dithering pattern consists of one pointing (stable spacecraft attitude) with $\mathrm{V} 0332+53$ positioned at the center of the field of view of the instruments, and six pointings with V $0332+53$ off-axis in steps of 2 degrees in a hexagonal pattern. The integration time for each pointing may vary from one observation to the next and is given in Table 1.

The V 0332+53 observations were affected by solar activity which impacted on the quality of the data. In particular, a strong solar flare lasting from Jan. 20 to 23 lead to a split of the IBIS data of revolution 278 into 7760 small Good Time Intervals (GTIs) of only few seconds each. The JEM-X GTIs were greater than $97 \%$ of the observation time for that revolution, and in general greater than $90 \%$ for all revolutions (see Table 1).

\section{Data analysis}

We analyse all the public data collected by the ISGRI and the JEM-X1 instruments on board of INTEGRAL. PICsIT is not useful for our analysis because the spectrum of V0332+53 falls off above $100 \mathrm{keV}$, and SPI data are not used either since its sensitivity in the 20 to $100 \mathrm{keV}$ range is less favorable than ISGRI (Paper I). Pointings close to perigee passage times are further discarded from the analysis as they are contaminated by the Earth radiation belt.

The ISGRI and JEM-X data are processed with version 5.0 of the Offline Scientific Analysis (OSA) package delivered by the INTEGRAL Science Data Center (http://isdc.unige.ch) using default settings. Images and lightcurves are produced in the $3-5 \mathrm{keV}, 5-10 \mathrm{keV}$ and $10-15 \mathrm{keV}$ energy bands for JEM-X, and in the $20-30 \mathrm{keV}$, $30-40 \mathrm{keV}$ and $40-60 \mathrm{keV}$ bands for ISGRI.

Contamination from other sources is not a problem as $\mathrm{V} 0332+53$ is the only bright X-ray source in the ISGRI field of view. Systematic errors are however known to affect the data analysis. In order to estimate the amplitude of those systematic errors, and to calibrate the results, an analysis of the Crab was performed with OSA parameters identical to those used for the analysis of V $0332+53$. Crab public data are taken from revolution 239, pointings 3 to 27 (Modified Julian Day MJD = 53275.5289 to 53276.1326), during which the Crab is located within 3 degrees from the center of the field of view. The resulting mean Crab fluxes extracted from the images in the $3-5,5-10,10-15 \mathrm{keV}$ bands of JEM-X are $43.5 \mathrm{cps}$ (14\% error), $43.2 \mathrm{cps}(10 \%)$ and $16.4 \mathrm{cps}$ (12\%), respectively. The quoted errors represent the maximum deviations measured from the mean fluxes over the 25 pointings. The Crab fluxes in the 20-30, 30-40 and 40-60 keV energy bands of ISGRI are $78.8 \mathrm{cps}(13 \%), 39.4 \mathrm{cps}$ (7\%) and $45.9 \mathrm{cps}(4 \%)$, respectively.

\section{Results}

\subsection{Fluxes}

The fluxes of V0332+53 in the ISGRI and JEM-X energy bands are plotted in Fig. 1 as a function of time. They are normalised to the Crab fluxes in the respective bands.

The decline phase can be described by an exponential decay up to $\mathrm{MJD} \simeq 53412$, followed by a linear decrease. 


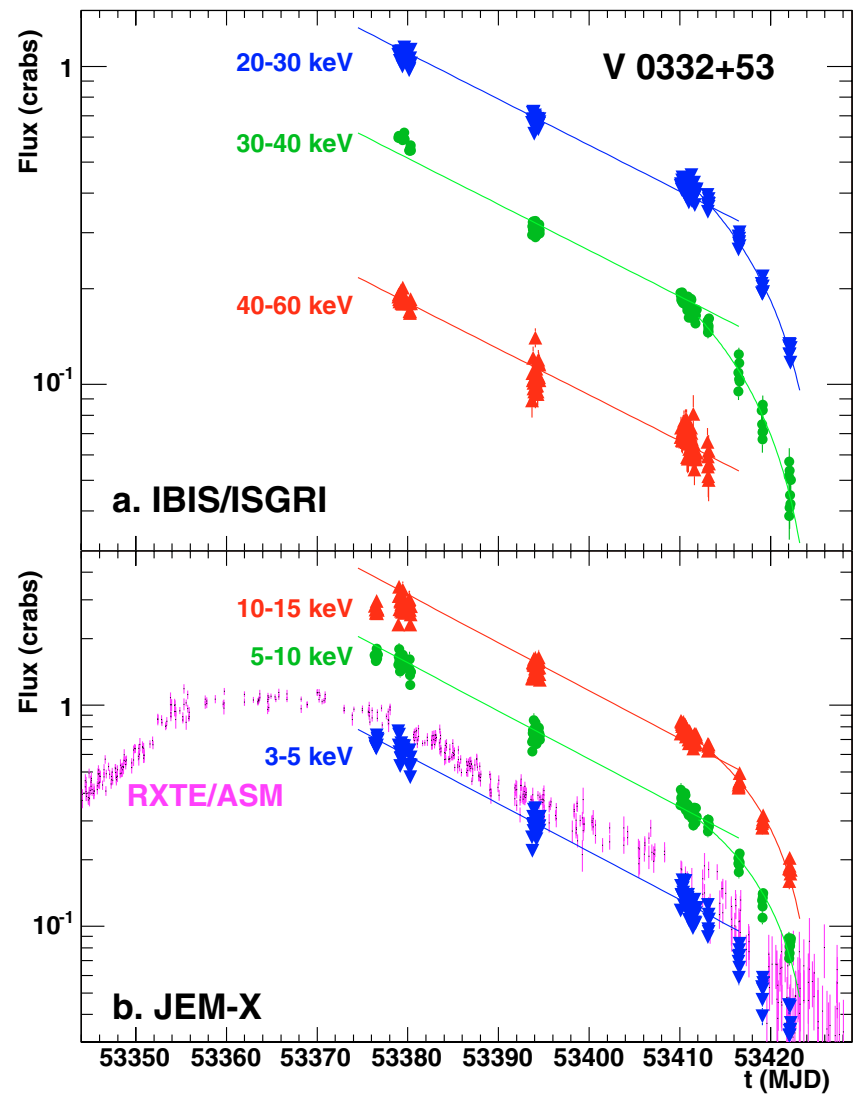

Fig. 1. a) Pointing averaged ISGRI flux evolution of V $0332+53$ as a function of time in the different energy bands as labelled in the figure. The fluxes have been normalised to Crab units. The error bars displayed in the figure represent only the statistical errors; neither do they take into account the uncertainties in the Crab fluxes in the respective energy bands. The solid curves are exponential (from MJD $=53376$ to 53412 with $\tau=30$ days) and linear (from MJD $=53412$ to 53422 ) decays superimposed on the data. b) Same as a), but for JEM-X fluxes and with an exponential decay of $\tau=20$ days for the solid curves. The 2-12 keV RXTE/ASM lightcurve is also shown, with a normalisation factor of $77 \mathrm{cps}$ for $1 \mathrm{Crab}$.

The exponential decay is less rapid at the ISGRI energies than at the JEM-X energies. From a simple estimation, the exponential decay time is about 30 days above $20 \mathrm{keV}$ (solid curves in Fig. 1a) and about 20 days below $15 \mathrm{keV}$ (solid curves in Fig. 1b). The exponential decay is also confirmed by the 2-12 keV lightcurve recorded by the All Sky Monitor (ASM) on board of RXTE, plotted in Fig. 1b for comparison with the JEM-X lightcurves (see Levine et al. 1996 for more information on the RXTE satellite).

The evolution of the ISGRI and JEM-X hardness ratios are shown in Fig. 2. Above $20 \mathrm{keV}$ the spectrum of $\mathrm{V} 0332+53$ softens with time, with the [30-40] keV / [20-30] keV ratio decreasing by about $36 \%$ from an average value of $\sim 0.55$ in the first observation to $\sim 0.35$ in the last observations. On the other hand, below $15 \mathrm{keV}$, the spectrum becomes harder, with the [10-15] keV / [5-10] keV ratio increasing by about $28 \%$ from an average value of $\sim 1.8$ to $\sim 2.3$ during the decline. As will be seen in Sect. 4.2, the opposite behaviour of the hardness ratios

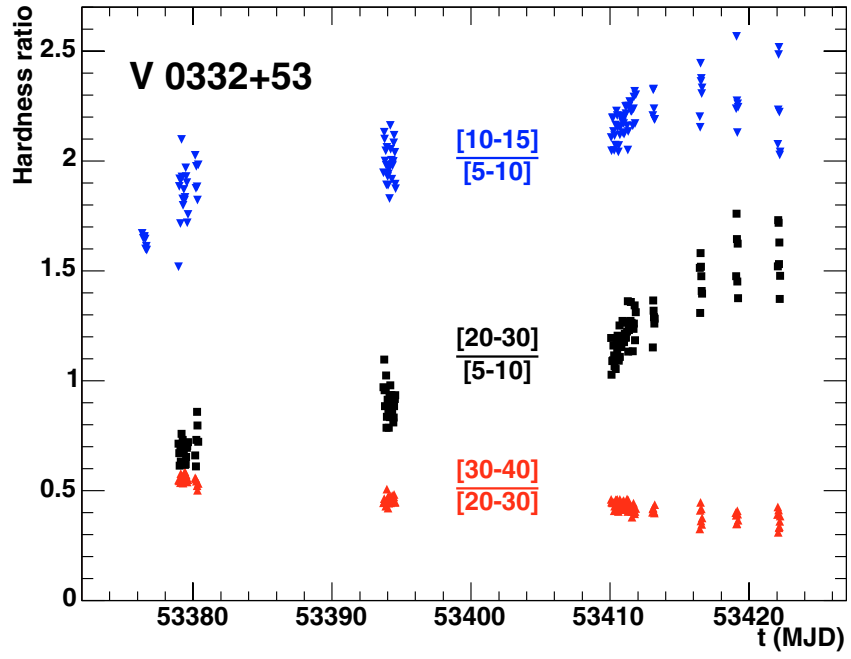

Fig. 2. Hardness ratios computed from the pointing-averaged (and Crab normalized) fluxes displayed in Fig. 1. Upper triangles: ISGRI [30-40 keV]/[20-30 keV]; lower triangles: JEM-X [10-15 keV]/ [5-10 keV]; squares: ISGRI [20-30 keV] / JEM-X [5-10 keV].

above $20 \mathrm{keV}$ and below $15 \mathrm{keV}$ are understood from the evolution of the spectral shape of $\mathrm{V} 0332+53$ during the decline.

The ISGRI/JEM-X hardness ratio ([20-30] keV/ [5-15] keV) also evolves with time, increasing from an average of $\sim 0.7$ to $\sim 1.5$ during the decline. This results from the increase of the spectral power law index presented in the next section.

\subsection{Energy spectra}

The JEM-X and ISGRI spectra of V $0332+53$, normalised to the Crab spectrum, are shown in Fig. 3 at four different times during the decline of the source. The maximum flux relative to the $\mathrm{Crab}$ is recorded between 10 and $20 \mathrm{keV}$, at an energy $E_{\max }$ which increases with time. Fitting Gaussian curves to the JEM-X spectra in a $10 \mathrm{keV}$ width range around the maximum leads to $E_{\max }=14.6,15.4,16.8$ and $18.0 \mathrm{keV}$ for revolutions 273, 278, 284 and 287, respectively. This shift of $E_{\max }$ explains the hardening (softening) at low (high) energies noticed from the JEM-X (ISGRI) hardness ratios presented in Sect. 4.1.

The spectra of accreting X-ray pulsars include contributions from at least an accretion column and from the hotspots at the surface of the neutron star. In the absence of a consistent model, various empirical models are commonly used in the literature to fit such spectra (see for example Kreykenbohm et al. 1999 for a discussion). In this study, we use the simple "cutoffpl" model of the form

$F(E)=A \cdot E^{-\Gamma} \cdot \mathrm{e}^{-E / E_{\text {fold }}}$

to fit the combined ISGRI and JEM-X data, to which we add two cyclotron lines (see below). A multiplicative constant is applied to allow for the different normalisations of the two instruments. Furthermore a systematic error of $2 \%$ is applied to account for the uncertainties in the response matrices of JEM-X and ISGRI. No photoelectric absorption is required at low energies, compatible with the fact that we analyse the JEM-X data 
Table 2. Fitting parameters. From first to last column: (1) revolution number (see Table 1); (2) normalisation factor; (3) powerlaw index; (4) folding energy (cutoffpl in XSPEC); (5) energy of the fundamental cyclotron line; (6) width of the line; (7) optical depth at the line center; (8), (9) and (10) same as 5th, 6th and 7th columns, respectively, but for the first harmonic cyclotron line; (11) flux in the 2-10 keV band; (12) flux in the $20-40 \mathrm{keV}$ band.

\begin{tabular}{cccccccccccc}
\hline \hline Rev. & $A$ & $\Gamma$ & $\begin{array}{c}E_{\text {fold }} \\
\mathrm{keV}\end{array}$ & $\begin{array}{c}E_{\mathrm{cycl}, 1} \\
\mathrm{keV}\end{array}$ & $\begin{array}{c}\sigma_{1} \\
\mathrm{keV}\end{array}$ & $\begin{array}{c}\tau_{1} \\
E_{\mathrm{cycl}, 2} \\
\mathrm{keV}\end{array}$ & $\begin{array}{c}\sigma_{2} \\
\mathrm{keV}\end{array}$ & $\begin{array}{c}\tau_{2} \\
2-10 \mathrm{keV} \\
\mathrm{erg} / \mathrm{cm}^{2} / \mathrm{s}\end{array}$ & $\begin{array}{c}20-40 \mathrm{keV} \\
\mathrm{erg} / \mathrm{cm}^{2} / \mathrm{s}\end{array}$ \\
\hline 273 & $0.458_{-0.002}^{+0.004}$ & $-0.174_{-0.004}^{+0.005}$ & $7.04_{-0.02}^{+0.01}$ & $27.53_{-0.04}^{+0.04}$ & $4.70_{-0.03}^{+0.03}$ & $1.80_{-0.01}^{+0.01}$ & $51.5_{-0.1}^{+0.3}$ & $9.6_{-0.2}^{+0.1}$ & $1.82_{-0.02}^{+0.02}$ & $1.89 \times 10^{-8}$ & $7.31 \times 10^{-9}$ \\
274 & $0.35_{-0.00}^{+0.01}$ & $-0.26_{-0.02}^{+0.02}$ & $6.9_{-0.1}^{+0.1}$ & $27.7_{-0.1}^{+0.1}$ & $4.7_{-0.1}^{+0.1}$ & $1.78_{-0.04}^{+0.01}$ & $55.0_{-0.2}^{+1.3}$ & $11.3_{-0.6}^{+1.3}$ & $2.0_{-0.1}^{+0.1}$ & $1.66 \times 10^{-8}$ & $6.92 \times 10^{-9}$ \\
278 & $0.17_{-0.00}^{+0.01}$ & $-0.24_{-0.07}^{+0.02}$ & $7.5_{-0.3}^{+0.3}$ & $28.8_{-0.1}^{+0.1}$ & $4.8_{-0.1}^{+0.1}$ & $1.92_{-0.03}^{+0.04}$ & $54.6_{-0.8}^{+1.1}$ & $9.7_{-0.5}^{+0.7}$ & $2.6_{-0.1}^{+0.3}$ & $8.53 \times 10^{-9}$ & $4.37 \times 10^{-9}$ \\
284 & $0.06_{-0.00}^{+0.00}$ & $-0.41_{-0.01}^{+0.00}$ & $7.4_{-0.0}^{+0.0}$ & $29.3_{-0.0}^{+0.0}$ & $4.3_{-0.0}^{+0.0}$ & $1.96_{-0.02}^{+0.02}$ & $51.0 \mathrm{fix}$ & $7.4_{-0.1}^{+0.1}$ & $2.0_{-0.1}^{+0.1}$ & $3.94 \times 10^{-9}$ & $2.69 \times 10^{-9}$ \\
285 & $0.04_{-0.00}^{+0.00}$ & $-0.53_{-0.03}^{+0.01}$ & $7.2_{-0.3}^{+0.0}$ & $29.4_{-0.1}^{+0.1}$ & $4.4_{-0.1}^{+0.0}$ & $1.96_{-0.04}^{+0.04}$ & $51.0 \mathrm{fix}$ & $7.9_{-0.2}^{+0.2}$ & $2.1_{-0.1}^{+0.1}$ & $3.15 \times 10^{-9}$ & $2.41 \times 10^{-9}$ \\
286 & $0.03_{-0.00}^{+0.00}$ & $-0.44_{-0.06}^{+0.05}$ & $8.0_{-0.4}^{+0.2}$ & $29.5_{-0.1}^{+0.3}$ & $4.5_{-0.1}^{+0.1}$ & $2.00_{-0.05}^{+0.06}$ & $51.0 \mathrm{fix}$ & $7.9_{-0.8}^{+0.2}$ & $2.4_{-0.1}^{+0.2}$ & $2.19 \times 10^{-9}$ & $1.80 \times 10^{-9}$ \\
287 & $0.02_{-0.00}^{+0.00}$ & $-0.41_{-0.08}^{+0.05}$ & $8.3_{-0.5}^{+0.3}$ & $29.4_{-0.1}^{+0.3}$ & $4.1_{-0.1}^{+0.2}$ & $2.11_{-0.07}^{+0.08}$ & $51.0 \mathrm{fix}$ & $8.8_{-1.0}^{+0.4}$ & $2.7_{-0.2}^{+0.3}$ & $1.51 \times 10^{-9}$ & $1.28 \times 10^{-9}$ \\
\hline
\end{tabular}

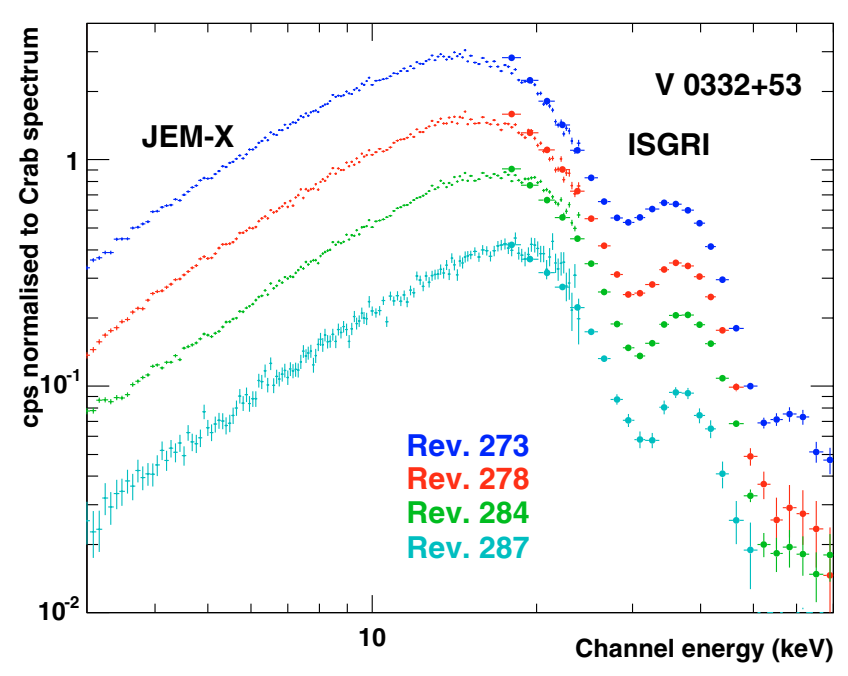

Fig. 3. JEM-X and ISGRI spectra integrated, from upper to lower curves, during revolutions 273, 278, 284 and 287, respectively. The spectra have been normalised to the Crab spectrum of revolution 239 (see text for details).

only above $4 \mathrm{keV}$; the addition of a $\mathrm{Fe} \mathrm{K} \alpha$ fluorescence line does not improve the fits either.

To fit the cyclotron lines we use Gaussian optical depth profiles of the form

$G_{i}(E)=\tau_{i} \cdot \mathrm{e}^{-\frac{1}{2}\left(\frac{E-E_{\mathrm{cycl}, i}}{\sigma_{i}}\right)^{2}}$

where $E_{\text {cycl }}$ is the line energy, $\sigma$ the line width, and $\tau$ the optical depth at the line center (see Kreykenbohm et al. 2004; Pottschmidt et al. 2005). The index $i$ identifies the line, i.e. $i=1$ for the fundamental at $28 \mathrm{keV}$ and $i=2$ for the first harmonic at $50 \mathrm{keV}$. The second harmonic at $71 \mathrm{keV}$ (Paper I) is not modelled due to insufficient statistics for revolution averaged spectra. The overall spectral model is then given by

$f(E)=F(E) \cdot \mathrm{e}^{-G_{1}(E)} \cdot \mathrm{e}^{-G_{2}(E)}$

where $F(E)$ is given by Eq. (1) and $G_{1}(E)$ and $G_{2}(E)$ by Eq. (2). The same model is used for all observations to keep consistency between the parameters from one observation to the next. The

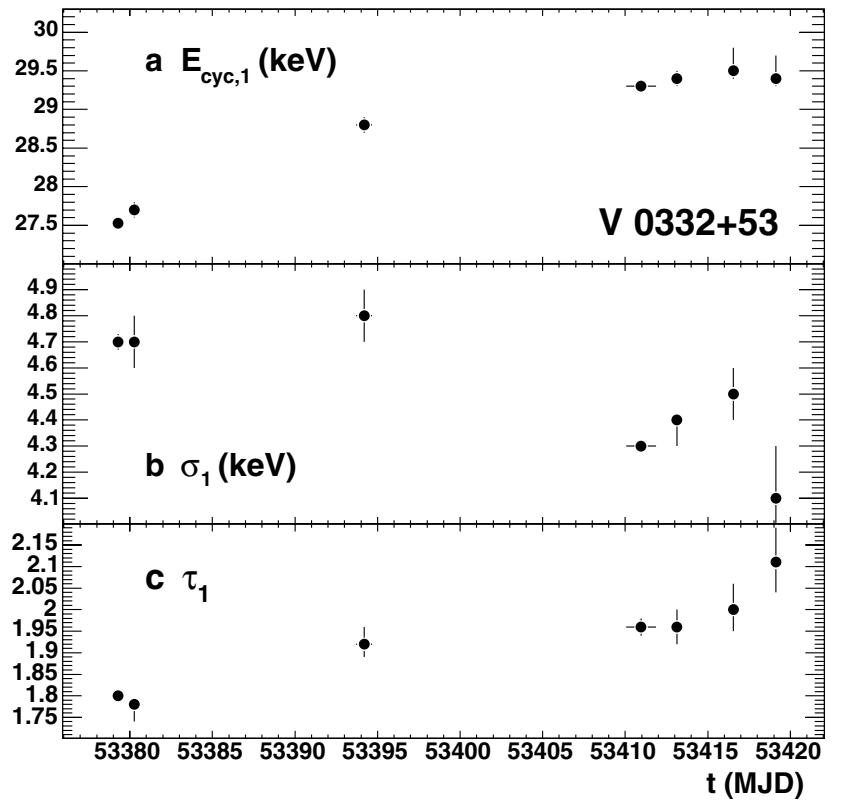

Fig. 4. Evolution of the specral fit parameters describing the fundamental cyclotron line. a) Line energy, b) width and c) depth. The horizontal bars on the points indicate the duration of each observation.

last observation (revolution 288) is disregarded from this spectral analysis due to poor statistics.

The main fit parameters are summarised in Table 2. The $2-10 \mathrm{keV}$ and $20-40 \mathrm{keV}$ fluxes derived from the fitted models confirm the two phases set forth in Sect. 4.1, i.e. first an exponential decay followed by a linear decrease of the flux. The folding energy remains about constant around $7.5 \mathrm{keV}$, which is consistent with the results of Paper I (Table 1) for revolution 273-274. The power law index $\Gamma$, on the other hand, decreases from -0.18 in the early revolutions to -0.4 in the later ones, in agreement with the hardening observed from the hardness ratios (Fig. 2).

The cyclotron line parameters for the fundamental are displayed in Fig. 4 as a function of time, and an example of the unfolded spectrum with the fit and residuals for revolution 284 is shown in Fig. 5. The energy of the fundamental cyclotron 


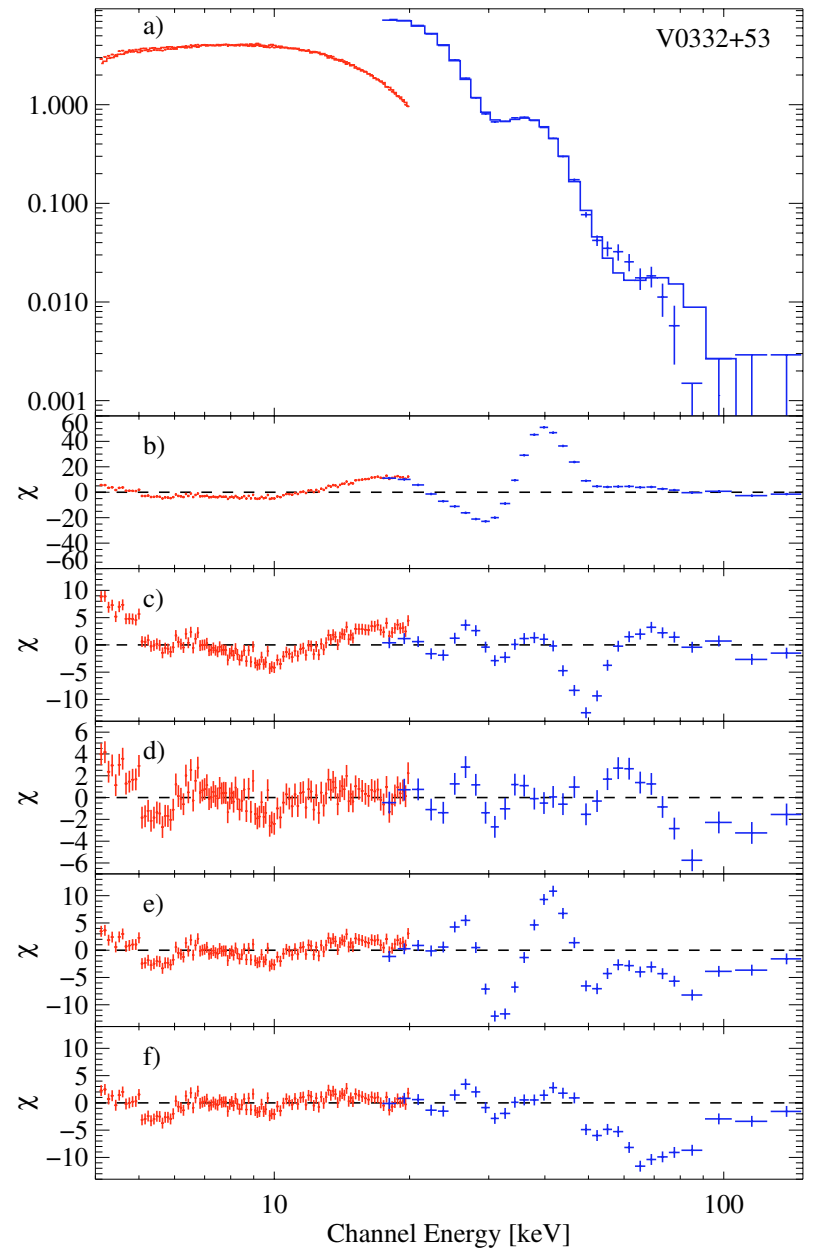

Fig. 5. a) Combined spectrum and folded model of data obtained with JEM-X (left) and ISGRI (right) for revolution 284; b) residuals of the model with $\Gamma=-0.41$ and $E_{\text {fold }}=7.4 \mathrm{keV}$, and without any Gaussian line applied; c) one Gaussian line is included at $29.3 \mathrm{keV}$; d) a second Gaussian is added, at $51 \mathrm{keV}$; e) same as d), but with the first line fixed at its value of $27.5 \mathrm{keV}$ found in revolution 273 ; f) same as d), but with $\Gamma$ fixed the value of -0.17 found in revolution 273 .

line increases by $7 \%$ from the first to the last INTEGRAL observations, while the depth of the line increases by $16 \%$ and the width decreases by $12 \%$. The changes are significant; attempting to fit the spectrum in revolution 284 with a line at $27.5 \mathrm{keV}$ leads to strong residuals around the line (Fig. 5e). The changes in the line parameters cannot be attributed to variations of the continuum either; fixing $\Gamma$ in revolution 284 to its value of -0.17 from revolution 273 leads to strong residuals at energies above $45 \mathrm{keV}$ (Fig. 5f).

The determination of the parameters describing the $50 \mathrm{keV}$ line becomes problematic from revolution 284 on, due to poor statistics. Therefore, for those revolutions, we fix the line energy to the value $E_{\mathrm{cycl}, 2}=51 \mathrm{keV}$ obtained for Rev. 273 where the statistics are the best, and fit the width and depth of the line to obtain the best residals. The results are shown in Table 2. The width and depth of line are seen to be rather stable throughout the whole decay of the outburst, around $9 \mathrm{keV}$ and 2, respectively. The large error bars on the numbers result from the poor statistics.

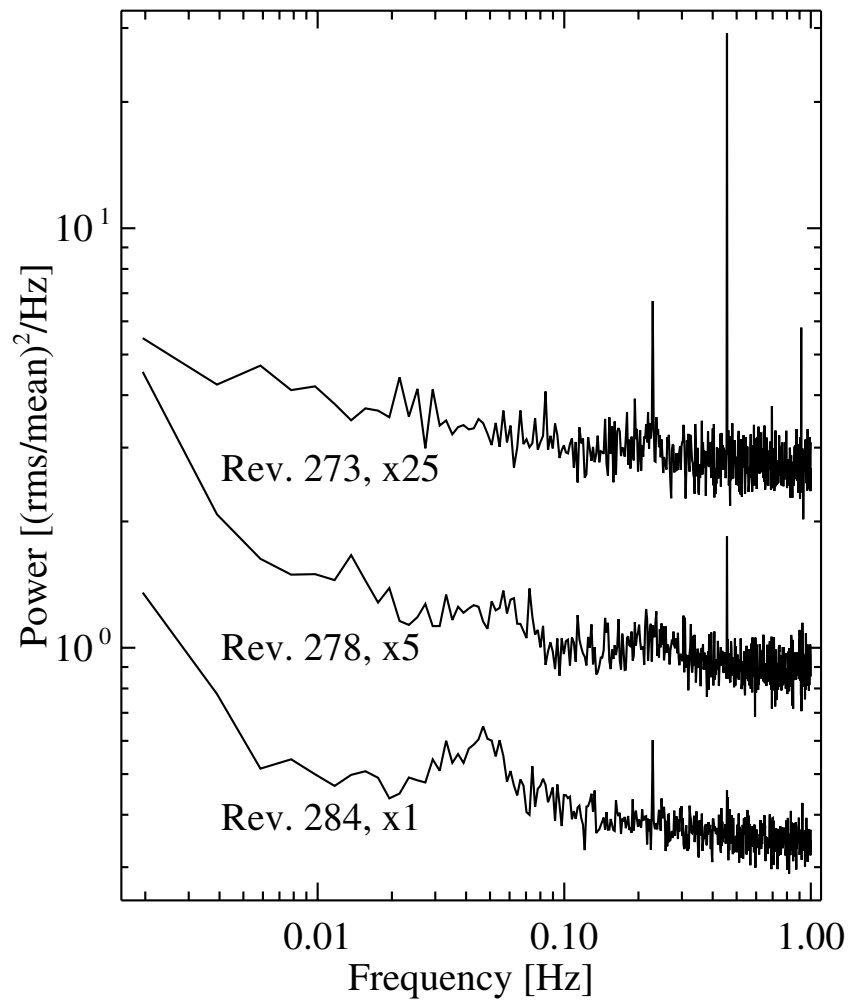

Fig. 6. 5-20 keV power spectra, averaged over the indicated revolutions and normalised using the method described by Miyamoto et al. (1991). Poisson noise has not been subtracted (see text for more details). The power spectra of revolutions 278 and 273 have been multiplied by factors of 5 and 25, respectively. Error bars have been omitted for clarity.

\subsection{Power spectra}

Several X-ray pulsars and cyclotron line sources are known to exhibit low frequency quasi-periodic oscillations (QPOs) (see, e.g., Takeshima et al. 1991; Takeshima et al. 1994; Heindl et al. 1999). Takeshima et al. (1994) first discovered a QPO with a centroid frequency of $\sim 0.05 \mathrm{~Hz}$ and a relative root mean square (rms) amplitude of $\sim 5 \%$ in $2.3-37.2 \mathrm{keV}$ data from the 1989 outburst of $\mathrm{V} 0332+53$. This feature has recently also been reported in the $2-60 \mathrm{keV} R X T E$-PCA power spectral density functions (PSDs) obtained during the 2004 outburst decay ( $\mathrm{Qu}$ et al. 2005). Furthermore Qu et al. found a $\mathrm{QPO}$ at $\sim 0.22 \mathrm{~Hz}$, i.e. centered on the fundamental pulsar frequency, in addition to the narrow peak at that frequency. The rms strength of this QPO also amounts to a few per cent. In the following we show that the JEM-X and ISGRI observations confirm the detection of both features during the outburst decay, and we analyse their changes with time. Note that, to our knowledge, these results represent the first QPOs reported from INTEGRAL JEM-X and ISGRI data in the literature.

The background subtracted $5-20 \mathrm{keV}$ and $20-40 \mathrm{keV}$ lightcurves of each revolution were rebinned to a time resolution of $0.5 \mathrm{~s}$ and power spectra were produced from $512 \mathrm{~s}$ long lightcurve segments using Fast Fourier Transforms. Averaged power spectra for selected revolutions are shown in Figs. 6 and 7. 


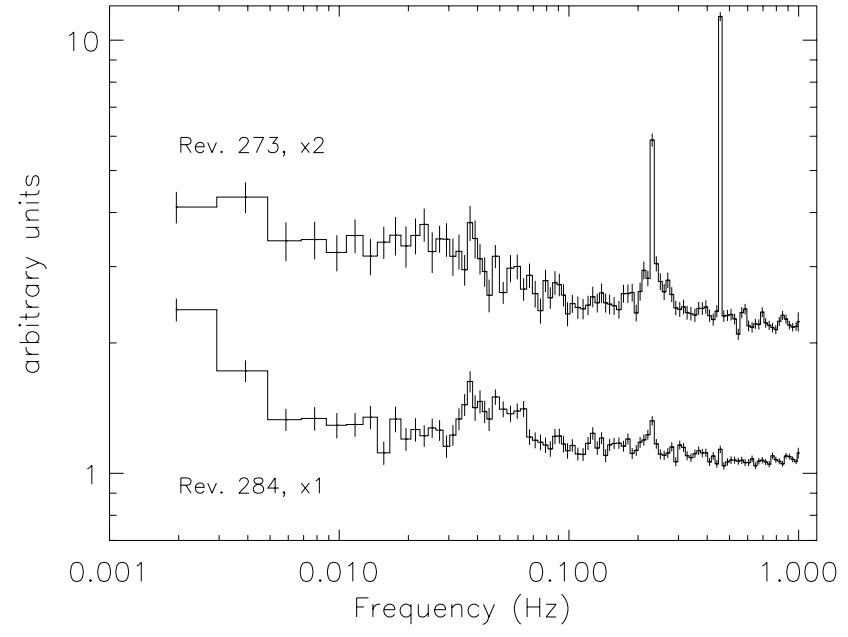

Fig. 7. 20-40 keV power spectra, averaged over the indicated revolutions. The spectra are arbitrarily shifted in the $y$-axis for easy comparison. Note that the ISGRI power spectra of revolution 278 are not shown due to solar flaring giving rise to many gaps in the lightcurve after GTI filtering.

For coded mask instruments the determination of the white noise level as well as the influence of the background flux on the power spectrum can be complex. The white noise component in JEM-X lightcurves, e.g., is known to be non-Poissonian. This is also true for the ISGRI which consists of 16000 pixels, each having different noise charactersistics. Using the normalization described by Leahy et al. (1983) we find the noise level to be about one order of magnitude higher than expected from Poisson noise. However, source features are well apparent above the noise level, e.g., the $0.05 \mathrm{~Hz}$ QPO is clearly visible in revolution 284. Therefore, and since the broad-band PSD (which is not to be discussed here) is dominated by the noise component up to at least $0.1 \mathrm{~Hz}$, we did not attempt to correct the power spectra for white noise. The JEM-X power spectra shown in Fig. 6 are normalised according to Miyamoto et al. (1991) and a comparison of the strength of features above the noise level between the JEM-X PSDs and the quasisimultaneous PCA PSDs of Qu et al. (2005) finds comparable values $^{1}$.

Strong changes in the shape of the power spectrum can be seen over the outburst decay, where the varying amplitude ratios at the pulsar frequency and its harmonics reflect a strong change of the pulse profile (Zhang et al. 2005). The PSDs shown in Figs. 6 and 7 represent the main stages of the evolution. Although, for the reasons discussed earlier, the white noise level is not accurately estimated in the INTEGRAL data, the JEM-X and ISGRI PSDs for revolutions 0273 and 0284 do compare well and show similar features as a function of frequency.

In revolution 273 (Jan. 8-9) the power spectrum is dominated by signatures of the pulsation frequency, especially a very strong first harmonic. In addition weak red noise is present.

\footnotetext{
${ }^{1}$ This can only be a very rough comparison since different energy bands are involved and since the power spectrum of V $0332+53$ obviously changes rapidly during the decay.
}

The peak at the fundamental pulsation frequency is slightly broadened at the bottom, especially in the ISGRI PSD. However, the $0.22 \mathrm{~Hz}$ QPO is not as obvious as a few days later in the Jan. 15-19 PCA power spectrum of Qu et al. (2005). In revolution 278 (Jan. 23-24) the fundamental pulsation at $0.22 \mathrm{~Hz}$ is not seen anymore but a weak broad QPO feature is present at this frequency. The first harmonic is still clearly visible. The strength of the red noise component more than doubled at low frequencies and there is an indication of additional power in the frequency region of the $0.05 \mathrm{~Hz}$ QPO. Principally the overall PSD shape is very similar to the Jan. 15-19 PCA power spectrum mentioned above. Finally, in revolution 284 (Feb. 9-10) the $0.05 \mathrm{~Hz}$ QPO is clearly visible, especially in the JEM-X power spectrum. The red noise is still strong while the pulsation fundamental is back as a narrow feature, stronger than the first harmonic. Fitting the low frequency QPO in revolution 284 with a Lorentzian gives $(0.049 \pm 0.001) \mathrm{Hz}$, with a FWHM of $(0.023 \pm 0.005) \mathrm{Hz}$ for JEM-X and $(0.049 \pm 0.003) \mathrm{Hz}$ with a FWHM of $(0.021 \pm$ $0.008) \mathrm{Hz}$ for ISGRI, where the underlying continuum can be modelled with a flat power law component and a very low frequency Lorentzian.

\section{Discussion}

$\mathrm{V} 0332+53$ is a highly magnetised pulsar which has a magnetic field of about $2.7 \times 10^{12} \mathrm{G}$ (Paper I, Pottschmidt et al. 2005) and orbits a massive O8-9Ve companion with a period of about 34 days. The X-ray outbursts from this system are observed with a time recurrence of about 10 years. They are believed to be triggered by a sudden increase of the mass ejection rate from the massive star, the signature of which has been observed in the optical brightening of the system in 2004 (Goranskij \& Barsukova 2004). The ejected mass feeds an accretion disk around the pulsar, from which it is funneled by the magnetic field towards the polar caps and falls on the surface of the neutron star.

The analysis of the 2005 outburst presented in Sect. 4 provides new hints towards a better understanding of $\mathrm{V} 0332+53$ within this basic picture. The X-ray lightcurve (Sect. 4.1) displays a characteristic exponential decay with a time scale of 20 to 30 days, followed by a linear decrease. This feature may provide some information on the disk properties. The X-ray spectrum (Sect. 4.2) is well fitted by a folding power law modified by two cyclotron lines. The $27 \mathrm{keV}$ energy of the fundamental cyclotron line is shown to increase by $7 \%$ during the outburst decay and to become narrower by $12 \%$. At the same time, its depth increases by $16 \%$. This information would provide some insight into the resonance scattering region near the polar caps. Finally, the appearance of the $0.05 \mathrm{~Hz}$ QPO only from the middle of the decay on (Sect. 4.3) provides further information on the properties of the matter around the neutron star. Each of those observational facts are successively discussed in the following sections.

\subsection{Lightcurve}

An exponential decay of the flux is observed in dwarf novae and in soft X-ray transients on timescales from a few days for 
the former to several weeks for the latter (e.g. Lasota 1996; Tanaka \& Shibazaki 1996). King \& Ritter (1998) show how illuminated disks in low-mass X-ray binary stars can produce such an exponential decay on the time scales observed for the soft X-ray transients. In that picture, the central star illuminates the disk and keeps its temperature high. The disk empties proportionally to its mass, leading to an exponential decay of the emitted flux. They further show that the lightcurve would be characterised by a linear decrease at later times. The change to a linear regime is triggered by a change in the conditions in the disk. In low-mass X-ray binaries the change corresponds to the decrease of the disk temperature below the temperature of partial $\mathrm{H}$ ionisation, such that the high temperature cannot hold over the entire disk anymore. This scenario of X-ray irradiated accretion disks has subsequently been confirmed by numerical calculations (Dubus et al. 1999, 2001).

It is tempting to make the parallel with the results found for $\mathrm{V} 0332+53$. The source displays an exponentially decreasing flux followed by a linear decrease, similar to the diskirradiated systems. This is the first time, to our knowledge, that such a behaviour is observed in a high-mass X-ray binary. Since the X-ray flux is proportional to the mass accretion rate, this picture suggests that the accretion rate is proportional to the mass of the disk during the exponential decay phase. The transition to a linear decrease phase would be triggered by an as yet un-identified change of conditions in the disk.

The two different exponential decay times observed in the lightcurves of $\mathrm{V} 0332+53$, of 30 days above $20 \mathrm{keV}$ and 20 days below (Sect. 4.1) suggest the presence of at least two regions contributing to the $\mathrm{X}$-ray continuum of that source. The harder component would originate from the accretion column near the resonant scattering region, while the softer component would originate from a region located, for example, higher in the accretion column. The observed increase of the depth of the cyclotron line with time is also compatible with this scenario; the softer component decays more rapidly and hence its tail would contribute relatively less to the high energy part of the spectrum from which the photons are scattered by the electrons in the magnetic field. Basically, one time scale governs the rate of mass flow onto the neutron star through the disk, leading to the exponential decay followed by a linear decrease (see above); another time scale governs the spectral changes of the emission that may come from different heights within the accretion column.

\subsection{Spectrum}

Time evolution of cyclotron resonance line parameters has already been reported in the literature for some binary systems. Mihara et al. (1998) report a relation between the line energy and the X-ray luminosity for 5 pulsars observed by Ginga. The resonance energy of $4 \mathrm{U} 0115+53$, for example, increased by $40 \%$ as the luminosity decreased by a factor of 6 between 1990 and 1991. The 1990 outburst of V $0332+53$ is also reported in that paper, with a $10 \%$ decrease of the line energy from $30.0 \pm 0.5 \mathrm{keV}$ to $27.2 \pm 0.3 \mathrm{keV}$ as the Ginga count rate in the $3-37 \mathrm{keV}$ band increased by a factor of 1.6. These authors attribute the change of the line energy to that of the height of the scattering region in a dipole magnetic field, leading to a height change of $\sim 1.1 \mathrm{~km}$ for $4 \mathrm{U} 0115+53$ and $\sim 330 \mathrm{~m}$ for V $0332+53$. Kreykenbohm et al. (2004), on the other hand, report a variation of the cyclotron energy of GX 301-2 with the pulse phase of the pulsar, and attribute the change to different viewing angles of the accretion column where the line originates.

In the case of the 2005 outburst decay of V $0332+53$ analysed in this paper, the increase with time of the energy and depth of the cyclotron fundamental line, together with the decrease of its width can also be understood as resulting from the variation of the scattering region characteristics in a dipole magnetic field above the polar caps of the pulsar. It is interesting to note that Pottschmidt et al. (2005), analyzing the RXTE data of V $0332+53$ taken two weeks before the first INTEGRAL observation, derive $E_{1}=25.2 \mathrm{keV}$ for 2004 December 24-26, which confirms the trend of increasing line energy with time found in this paper. Let us thus assume that the region where the cyclotron resonance scattering takes place extends over some fraction of the accretion column. Then the region would cover some range of magnetic strengths, and a broad line shape would result from the superposition of a succession of narrow lines, each originating from a different height and magnetic field strength in the accretion column. As the accretion rate and hence the flux decreases with time, the extension of the resonance scattering region is expected to decrease along with the total width of the line. At the end of the decline only the region close to the neutron star surface, where the magnetic field is stronger, would contribute to the resonance scattering; this would lead to a narrower line shape at a higher energy. The observed energy $E_{1}$ indeed increases by $17 \%$ from the RXTE observation of December 2004 to the last INTEGRAL observation in February 2005. If we consider a dipole approximation for the magnetic field, we have $B \sim r^{-3}$ in a non-relativistic description, where $r$ is the radius to the center of the neutron star. The extent of the resonance scattering region at the peak of the outburst would then, in this first scenario, be about $6 \%$ of the stellar radius, i.e. about 500 meters for typical radii of neutron stars. A change in the scattering energy between two different observations of Her X-1, from $34 \mathrm{keV}$ to $41 \mathrm{keV}$, has been discussed by Gruber et al. (2001); they conclude that the range over which the scattering occurs is of the order of $1 \mathrm{~km}$ in height, if their observed line width of $5 \mathrm{keV}$ is largely due to a mix of magnetic field strengths in a dipole field. In the case of $\mathrm{V} 0332+53$, we additionally observe an evolution of the width and depth of the line with time.

Alternatively, we can argue that the fundamental line originates from a unique narrow region in the column density, but that the line shape is quite complex and variable with time, resulting in variations of the line width and depth. An increase in the line energy would then be related to a displacement with time of the scattering region. In the case of V $0332+53$, the observed $17 \%$ increase of $E_{1}$ would imply a move of the location of the scattering region by $500 \mathrm{~m}$ in the accretion column down to the surface of the neutron star. However, in this second scenario, the line profile is expected to remain approximately 
constant with time, which is not supported by the INTEGRAL observations. Also, if the scattering region gets closer to the neutron star, higher order terms in the B-field may increase the line width, which is opposite to the trend observed by INTEGRAL. We therefore would rather support the first scenario.

\subsection{Quasi periodic oscillations}

The $0.22 \mathrm{~Hz}$ QPO observed around the spin frequency in both the INTEGRAL (Sect. 4.3) and RXTE (Qu et al. 2005) data must originate from a region around the neutron star co-rotating with the pulsar. This region can either be located at the inner radius of the accretion disk near the Alfvén radius, or in the corona above the hot spots, or somewhere in between along the magnetic field through which the matter is tunneled.

The origin of the lower frequency QPO at $0.05 \mathrm{~Hz}$, on the other hand, is more debatable. Low frequency QPO-type variabilities are known in several X-ray pulsars (see van der Klis 2004 for a review). Although there is no general consensus on the origin of QPOs, most models do invoke the presence of some sort of inhomogeneities in the accretion disk. With a typical neutron star mass $M_{\mathrm{NS}}=1.4 M_{\odot}$ and a spin of $P_{\mathrm{NS}} \simeq 4 \mathrm{~s}$ for $\mathrm{V} 0332+53$, the co-rotation radius where the angular velocity of the magnetosphere and the Keplerian velocity of the disk are equal is

$R_{\text {corot }} \simeq 1500\left(\frac{M_{\mathrm{NS}}}{M_{\odot}}\right)^{1 / 3}\left(\frac{P_{\mathrm{NS}}}{\mathrm{s}}\right)^{2 / 3} \mathrm{~km} \simeq 4230 \mathrm{~km}$.

On the other hand, the radius at which the Keplerian frequency is equal to that of the $0.05 \mathrm{~Hz}$ QPO is $\sim 12350 \mathrm{~km}$. The scenario of having the $0.05 \mathrm{~Hz}$ QPO somehow related to the part of the disk at this distance, i.e. three times further away than the corotation radius, is thus plausible. The temperature of the disk cannot be responsible for the observed emission, since no black body emission is found in the spectrum. The QPO could result from an occultation of the beam by intervening matter from the disk at that distance, as suggested by Heindl et al. (1999) to explain a $2 \mathrm{mHz}$ QPO observed in 4U 0115+63.

\section{References}

Coburn, W., Kretschmar, P., Kreykenbohm, I., et al. 2005, ATel, 381, 1

Dubus, G., Lasota, J.-P., Hameury, J.-M., \& Charles, P. 1999, MNRAS, 303, 139

Dubus, G., Hameury, J.-M., \& Lasota, J.-P. 2001, A\&A, 373, 251

Goranskij, V., \& Barsukova, E. 2004, ATel, 245, 1

Gruber, D. E., Heindl, W. A., Rothschild, R. E., et al. 2001, ApJ, 562, 499

Heindl, W. A., Coburn, W., Gruber, D. E., et al. 1999, ApJ, 521, L49

King, A. R., \& Ritter, H. 1998, MNRAS, 293, L42

Kreykenbohm, I., Kretschmar, P., Wilms, J., et al. 1999, A\&A, 341, 141

Kreykenbohm, I., Wilms, J., Coburn, W., et al. 2004, A\&A, 427, 975

Kreykenbohm, I., Mowlavi, N., Produit, N., et al. 2005, A\&A, 433, L45 (Paper I)

Lasota, J. P. 1996, IAUS, 163, 43

Leahy, D. A., Darbro, W., Elsner, R. F., et al. 1983, ApJ, 266, 160

Levine, A. M., Bradt, H., Cui, W., et al. 1996, ApJ, 469, L33

Mihara, T., Makishima, K., \& Nagase, F. 1998, Adv. Space Res., 22, 987

Miyamoto, S., Kimura, K., Kitamoto, S., et al. 1991, ApJ, 383, 784

Pottschmidt, K., Kreykenbohm, I., Wilms, J., et al. 2005, ApJ, 634, L97

Qu, J., Zhang, S., Song, L., \& Falanga, M. 2005, ApJ, 629, L33

Swank, J., Remillard, R., \& Smith, E. 2004, ATel, 349

Tanaka, Y., \& Shibazaki, N. 1996, ARA\&A, 34, 607

Takeshima, T., Dotani, T., Mitsuda, K., \& Nagase, F. 1991, PASJ, 43, L43

Takeshima, T., Dotani, T., Mitsuda, K., \& Nagase, F. 1994, ApJ, 436, 871

van der Klis, M. 2004, in Compact stellar X-ray sources, ed. Lewin \& van der Klis (Cambridge University Press) [arXiv: astro-ph/0410551]

Winkler, C., Courvoisier, T. J.-L., Di Cocco, G., et al. 2003, A\&A, 411, L1

Zhang, S., Qu, J. L., Song, L. M., \& Torres, D. F. 2005, ApJ, 630, L65 